Pacific

Journal of

Mathematics

\title{
SUPERDISTRIBUTIONS, ANALYTIC AND ALGEBRAIC SUPER HARISH-CHANDRA PAIRS
}

Claudio Carmeli and Rita Fioresi 


\title{
SUPERDISTRIBUTIONS, ANALYTIC AND ALGEBRAIC SUPER HARISH-CHANDRA PAIRS
}

\author{
Claudio CARMEli AND Rita Fioresi
}

\begin{abstract}
We extend the theory of super Harish-Chandra pairs, originally developed by Kostant and Koszul for smooth Lie supergroups, to algebraic supergroups over a field of characteristic zero. We also review the corresponding complex analytic theory and we give a characterization of the action of an algebraic (resp. complex analytic) super Harish-Chandra pair on a supervariety (resp. complex analytic supermanifold).
\end{abstract}

\section{Introduction}

The main purpose of this paper is to extend the theory of super Harish-Chandra pairs, originally developed by Kostant [1977] and Koszul [1983] for smooth Lie supergroups, to algebraic supergroups, enlightening similarities and differences with the complex analytic setting, treated in detail by Vishnyakova [2011]. This approach appears to be especially fruitful in the study of algebraic supergroup representations and more in general supergroup actions on supervarieties.

Roughly speaking, a super Harish-Chandra pair (SHCP for short) consists of a pair $\left(G_{0}, \mathfrak{g}\right)$, where $G_{0}$ is an ordinary algebraic (resp. analytic or smooth) supergroup and $\mathfrak{g}$ is a Lie superalgebra, with even part $\mathfrak{g}_{0}=\operatorname{Lie}\left(G_{0}\right)$. If $G$ is a supergroup (algebraic, analytic or differential), we have a natural SHCP associated with it: $\left(G_{0}, \operatorname{Lie}(G)\right)$. What appears to be surprising is the fact that the correspondence between supergroups and SHCP is bijective (up to isomorphism), i.e., starting from a given $\operatorname{SHCP}\left(G_{0}, \mathfrak{g}\right)$, we can reconstruct a supergroup, which has a corresponding $\operatorname{SHCP}\left(G_{0}, \operatorname{Lie}(G)\right)=\left(G_{0}, \mathfrak{g}\right)$, and such supergroup is unique. Actually more is true: there is an equivalence of categories between the category of supergroups (algebraic, analytic or differential) and the category of SHCPs (algebraic, analytic or differential), once morphisms are properly defined.

Such equivalence in the smooth context dates back to [Koszul 1983], while the analytic setting is due to Vishnyakova [2011], though a careful reading of [Koszul 1983], shows that the complex theory appeared already, somehow implicitly, in that

MSC2010: primary 14A22, 14M30, 32C11, 58A50; secondary 16S38, 58C50.

Keywords: supergroups, supergeometry. 
paper. Vishnyakova applied the result about the equivalence of categories between analytic supergroups and analytic SHCPs to provide a characterization of those complex homogeneous analytic supermanifolds that are split. We take her work a step forward: we characterize the concept of action of an analytic SHCP on an analytic supermanifold, proving it is equivalent to the ordinary notion of action of an analytic super Lie group on an analytic supermanifold. Our result, which is novel, immediately carries over to the affine algebraic category.

After our paper appeared on the web on June 2011, Masuoka [2012] published a more general and very interesting result in which he quoted our work, giving us the credit for being the first authors to treat the algebraic setting for the equivalence of categories between algebraic supergroups and algebraic SHCPs in characteristic zero. Masuoka is able to obtain a generalization of our result through a characteristic free approach, in purely algebraic terms.

In his paper, Masuoka defines a category of SHCPs whose objects are pairs consisting of an Hopf algebra $C$ and a finite dimensional right $C$-comodule $W$, together with appropriate compatibility conditions. In the characteristic zero case, the category of Masuoka's SHCPs is anti-isomorphic to the algebraic SHCP category we use in the present paper. He then establishes an equivalence between the category of such SHCPs $(C, W)$ and the category of affine (i.e., super commutative and finitely generated) Hopf superalgebras, which in turn is contravariantly equivalent to the category of affine algebraic supergroups. The functor establishing such an equivalence associates to each pair $(C, W)$ a subalgebra $A(C, W)$ of the completion of the smash product Hopf algebra $C \times^{\prime} T(W)$ (here $T(W)$ denotes the tensor algebra of $W$ ). In this sense, Masuoka's approach seems more related to Kostant's proof of the categorical equivalence between smooth SHCPs and smooth super Lie groups. Indeed in his approach Kostant realizes the structure sheaf of the supergroup as a subalgebra of the algebraic dual of the smash product $\mathbb{R}\left[G_{0}\right] \times^{\prime} \mathcal{U}(\mathfrak{g})$. We believe that the importance of Koszul's approach relies in the simple geometrical realization of the sheaf as the coinduced module

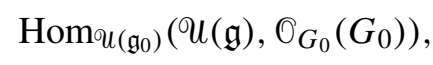

which is very explicit. This is particularly important when one tries to deduce general properties of super Lie groups (see, for instance, the characterization of split homogeneous supermanifold in [Vishnyakova 2011], or our Proposition 4.3). Moreover, as far as we understand, it is still an open problem to establish whether the correspondence between SHCPs as we define them and algebraic supergroups is an equivalence of categories in the positive characteristic case.

Since our methods are essentially different from Masuoka's and present a geometric point of view particularly useful for the applications (see our Section 4), we believe that our work still deserves a place in the literature. 
Our treatment begins with the definition of distribution superalgebra. We keep our discussion general enough to accommodate both the analytic and algebraic category and we believe this is one of the strengths of our paper and it singles it out from the previous treatments of the same subject we quoted above, which usually deal with just one category (algebraic, analytic or differential) at a time. The distribution superalgebra is a key object; its definition in differential supergeometry dates back to Kostant [1977], who first recognized its importance in this context. As we show in our work, the distribution superalgebra $D(G)$ of a supergroup $G$ (algebraic, analytic or differential) is naturally equipped with a Hopf superalgebra structure and it is indeed this Hopf structure, which makes possible the reconstruction of the algebraic, analytic or differentiable supergroup associated with an SHCP. In fact, when the characteristic of the ground field $k$ is zero, $D(G)$ is linearly isomorphic to $k|G| \otimes U(\mathfrak{g})(k|G|$ denoting the ordinary group algebra associated with the topological group $|G|$ underlying the supergroup $G$ ). This allows us to endow $k|G| \otimes U(\mathfrak{g})$ with an Hopf superalgebra structure, inherited by $D(G)$ via the above mentioned linear isomorphism. The superalgebra of the global sections of the structural sheaf of the algebraic supergroup $G$, associated (uniquely) with the given SHCP $(|G|, \mathfrak{g})$, is then realized inside the dual of $k|G| \otimes U(\mathfrak{g})$, thus inheriting its Hopf structure. This is essentially the reason why the above mentioned equivalence of categories works, though the proofs and the statements are necessarily more complicated, since of the technicalities involved, which at this point differ depending on the category we consider, for example for the analytic category we cannot take into consideration the global sections only, but we need to look at the whole sheaf.

This paper is organized as follows.

In Section 2 we describe the superalgebra of distributions of an analytic or an algebraic supergroup, establishing its relation with the universal enveloping superalgebra. The material exposed here is general common knowledge, though we are not aware of a treatment as complete and general as ours.

Section 3 contains the main results of our paper, including Theorem 3.6, which establishes the equivalence of categories between SHCPs (algebraic or analytic) and supergroups (algebraic or analytic). For the reader's convenience, this is preceded (starting on page 39) by a brief review of the equivalence between the category of analytic SHCPs and the category of analytic supergroups. ${ }^{1}$ Subsequently (page 42) we establish the equivalence between the category of algebraic SHCPs and the category of affine algebraic supergroups under suitable hypothesis for the ground field. The results of this section were generalized in [Masuoka 2012], with totally different methods, posted on the web at a later date than ours.

In Section 4 we provide an equivalent approach to the study of the actions of

${ }^{1}$ The material of this section appeared already, essentially in this form, in [Vishnyakova 2011]. 
supergroups, via SHCPs. This result extends the result stated in [Deligne and Morgan 1999] for the smooth category (see also [Balduzzi et al. 2009; Carmeli et al. 2011]). These results are novel as far as we know.

We believe the present work is justified, given the importance of the algebraic theory for practical purposes together with the lack of an appropriate and complete available reference.

For all the definitions and main results in supergeometry expressed with our notation, we refer the reader to [Fioresi and Gavarini 2011] or [Fioresi and Gavarini 2012, Chapter 2] or [Carmeli et al. 2011, Chapters 1, 4, 10]. In particular we shall employ both the sheaf-theoretic and the functor of points approach to supergeometry. On this we invite the reader to consult the classical references [Deligne and Morgan 1999; Manin 1988; Varadarajan 2004].

\section{The superalgebra of distributions}

We start by giving the definition of distribution and distribution superalgebra. Our treatment is general enough to accommodate the two very different categories of supermanifolds and superschemes. For the classical definitions we send the reader to [Jantzen 2003, page 95], [Demazure and Gabriel 1970, Chapter II §4, no. 6], and [Dieudonné 1970]. For the basic definitions of supergeometry we refer the reader to [Manin 1988; Varadarajan 2004; Deligne and Morgan 1999; Fioresi and Gavarini 2012].

Distributions. Let $k$ be the ground field.

Let $X=\left(|X|, O_{X}\right)$ be an analytic supermanifold or an algebraic superscheme over the field $k^{2}$

Let $X(k)$ be the $k$-points of $X$, that is $X(k)=\operatorname{Hom}\left(k^{0 \mid 0}, X\right)$ in the functor of points notation. For an analytic supermanifold $X$ we have that its $k$-points $X(k)$ are identified with the topological points $|X|$, while for $X$ a superscheme the $k$-points, are in one to one correspondence with the rational points, that is, the points $x \in|X|$ for which $\mathrm{O}_{X, x} / m_{X, x} \cong k, m_{X, x}$ being the maximal ideal in the stalk $\mathrm{O}_{X, x}$.

Definition 2.1. A distribution supported at $x \in X(k)$ of order at most $n$ is a morphism $\phi: \mathbb{O}_{X, x} \rightarrow k$, with $m_{X, x}^{n+1} \subset \operatorname{ker}(\phi)$ for some $n$. The set of all distributions at $x$ of order $n$ is denoted as $D_{n}(X, x)$, while $D(X, x)$ denotes all distributions supported at $x$. Both $D_{n}(X, x)$ and $D(X, x)$ have a natural super vector space structure.

We also define

$$
D(X)=\bigcup_{x \in X(k)} D(X, x)
$$

${ }^{2}$ If $X$ is an analytic supermanifold, $k=\mathbb{R}$ or $k=\mathbb{C}$ or even $k=\mathbb{Q}_{p}$, the $p$-adic numbers (see for example [Serre 1992]). If $X$ is a superscheme, $k$ is a generic field. 
as the distributions of finite order of $X$. Also $D(X)$ has a natural super vector space structure.

Observation 2.2. (1) We have

$$
D_{n}(X, x) \cong\left(\mathcal{O}_{X, x} / m_{X, x}^{n+1}\right)^{*},
$$

since if $\phi \in D_{n}(X, x)$, we have $\phi\left(m_{X, x}^{n+1}\right)=0$; hence $\phi$ factors and becomes an element in $\left(\mathcal{O}_{X, x} / m_{X, x}^{n+1}\right)^{*}$. Further notice that

$$
D_{0}(X, x)=k, \quad D_{1}(X, x)=k \oplus\left(m_{X, x} / m_{X, x}^{2}\right)^{*} .
$$

Hence $D_{1}(X, x)^{+}:=\left(m_{X, x} / m_{X, x}^{2}\right)^{*}$ becomes identified with the tangent space to $X$ at the point $x$.

(2) If $X$ is an affine algebraic superscheme, $O(X)$ the superalgebra of the global sections of its structural sheaf, a distribution supported at $x$ of order $n$ can be equivalently seen as a morphism $\phi: \mathbb{O}(X) \rightarrow k$, with $m_{x}^{n} \subset \operatorname{ker}(\phi)$, where $m_{x}:=$ $\{\phi \in \mathcal{O}(X) \mid \phi(x)=0\}$ is the maximal ideal of all the functions vanishing at $x$, where as usual in supergeometry $f(x)$ simply means the image in $\mathcal{O}_{X, x} / m_{X, x}$ of the element $f \in \mathcal{O}(X)$ under the natural morphisms: $\mathscr{O}(X) \rightarrow \mathcal{O}_{X, x} \rightarrow \mathcal{O}_{X, x} / m_{X, x} \cong k$. (Notice that since $x$ is rational, we have $\mathcal{O}(X)=k \oplus m_{x}$ and $\mathcal{O}_{X, x} / m_{X, x} \cong k$ ).

We leave it to the reader to check that the two definitions of distributions given are essentially the same in this case.

(3) If $X$ is a smooth supermanifold, that is, if we are in the differential category, we can view a point supported distribution as a morphism $\phi: \mathbb{O}(X) \rightarrow \mathbb{R}, m_{x}^{n} \subset \operatorname{ker}(\phi)$, where $m_{x}$ is the maximal ideal corresponding to the point $x \in|X|$ (see [Kostant 1977] and [Carmeli et al. 2011, 4.7]), thus recovering the same definition as in (2) for the affine algebraic category. This is one of the many analogies between the category of affine supervarieties and smooth supermanifolds.

Example 2.3 (distributions on $k^{p \mid q}$ ). Here we assume $\operatorname{char}(k)=0$. Consider the superspace $X=k^{p \mid q}$ (both in the analytic and affine algebraic context). Let $x_{1} \ldots x_{p}$, $\xi_{1} \ldots \xi_{q}$ denote the global coordinates and $m_{0}=\left(x_{1} \ldots x_{p}, \xi_{1} \ldots \xi_{q}\right)$ the maximal ideal in the stalk $\mathrm{O}_{X, 0}$ at the origin. We have

$$
\mathrm{O}_{X, 0} / m_{0}^{n+1} \cong \operatorname{span}_{k}\left\{1, x_{1}^{i_{1}} \ldots x_{p}^{i_{p}} \xi_{1}^{i_{p+1}} \ldots \xi_{q}^{i_{p+q}}, \sum i_{k}=n\right\} .
$$

If $I=\left(i_{1} \ldots i_{p+q}\right)$, let $X^{I}$ denote the monomial $x_{1}^{i_{1}} \ldots x_{p}^{i_{p}} \xi_{1}^{i_{p+1}} \ldots \xi_{q}^{i_{p+q}}$. Since the distributions at 0 of order $n$ are the dual of the super vector space $0_{X, 0} / m_{0}^{n+1}$, we have that a basis for the super vector space of distributions at the point 0 is given by $\phi_{J}$ such that $\phi_{J}\left(X_{I}\right)=\delta_{I J}$, with $I=\left(i_{1} \ldots i_{p+q}\right), J=\left(j_{1} \ldots j_{p+q}\right)$ multiindices, 
$\sum i_{k}=\sum j_{k}=n$. So we have

$\phi_{j_{1} \ldots j_{p+q}}(f)=\frac{1}{j_{1} ! \ldots j_{p+q} !}\left(\frac{\partial}{\partial x_{1}}\right)^{j_{1}} \ldots\left(\frac{\partial}{\partial x_{p}}\right)^{j_{p}}\left(\frac{\partial}{\partial \xi_{q}}\right)^{j_{p+1}} \ldots\left(\frac{\partial}{\partial \xi_{1}}\right)^{j_{p+q}}(f)(0)$.

The superalgebra of distributions of an analytic supermanifold. In this section we characterize the distributions for an analytic supermanifold $M=\left(|M|, O_{M}\right)$ in the following way. Distributions at the point $x \in|M|$ are the elements in $0_{M, x}^{*}$ whose kernel contains an ideal of finite codimension, in analogy with Kostant's treatment [1977] for the smooth category. We start with a lemma.

Lemma 2.4. Let $M=\left(|M|, O_{M}\right)$ be an analytic supermanifold, $x \in|M|, m_{X, x}$ the ideal in $\mathrm{O}_{M, x}$ of the sections vanishing at $x$. For each positive integer $p, m_{X, x}^{p}$ is an ideal of finite codimension.

Proof. It follows from the Taylor expansion formula. In fact, every element $f$ in $\mathcal{O}_{M, x}$ can be written as $f=\sum_{I} f_{I} \theta^{I}$, where $f_{I}$ is an element in the classical stalk of germs of holomorphic functions $\mathscr{H}_{M, x}$. For each positive integer $q$, a germ $f_{I}$ can in turn be written as

$$
f_{I}(z)=f_{I}(x)+\sum_{K: 1 \leq|K| \leq q-1}\left(\partial_{K} f_{I}\right)(x) z^{K}+\sum_{J:|J|=q} z^{J} h_{I, J}(z)
$$

where $I, J, K$ are multiindices. Hence we can write

$$
f=\sum_{I}\left(f_{I}(x)+\sum_{R:|R+I|<p}\left(\partial_{R} f_{I}\right)(x) z^{R}\right) \theta^{I}+\sum_{|I+R|=p} h_{I, R}(z) z^{R} \theta^{I} .
$$

From this formula, it follows that the elements in $m_{X, x}^{p}$ are generated by the monomials $\left\{z^{K} \theta^{I}\right\}_{|K+I| \leq p}$, and $\mathcal{O}_{M, x} / m_{M, x}^{p}$ has finite dimension.

Proposition 2.5. An ideal $J$ in $\mathrm{O}_{M, x}$ has finite codimension if and only if there exists an integer $p>0$ such that $m_{M, x}^{p} \subseteq J$.

Proof. The "if" part follows from the previous lemma. For the "only if" part we reason as follows. Consider the descending chain of ideals $J+m_{M, x}^{p} \supseteq J+m_{M, x}^{p+1}$. Since $J$ has finite codimension there exists $q$ such that $J+m_{M, x}^{q}=J+m_{M, x}^{q+1}$. From this it follows that $m_{M, x}^{q} \subseteq J+m_{M, x}^{q} \cdot m_{M, x}$. Since, by the previous lemma, $m_{M, x}^{q}$ is finitely generated we can apply the super version of Nakayama lemma (see [Varadarajan 2004]) and we get $m_{M, x}^{q} \subseteq J$.

We have then obtained the following result, which establishes a parallelism with the smooth category.

Theorem 2.6. The distributions on an analytic supermanifold $M$ supported at a point $x$ correspond to morphisms $f: \mathrm{O}_{M, x} \rightarrow k$ whose kernel contains an ideal of finite codimension. 
The distributions of a supergroup at the identity. We now want to restrict our attention to the distributions of a supergroup (analytic or algebraic) at the identity element $e \in G(k)$.

As a consequence of the Observation 2.2, we have

$$
D_{1}(G, e)^{+} \cong\left(m_{G, e} / m_{G, e}^{2}\right)^{*} \cong T_{e}(G)=\operatorname{Lie}(G) .
$$

It is only natural to expect $D(G, e)$ to be identified with $U(\mathfrak{g})$, with $\mathfrak{g}=\operatorname{Lie}(G)$. This is true, as we shall see, provided we exert some care.

As we remarked in the Definition 2.1 the distributions at the identity are a super vector space, however there is a natural additional superalgebra structure that we can associate to the super vector space of distributions, by defining the convolution product.

Definition 2.7. Let $\phi, \psi \in D(G, e)$. We define their convolution product as the following morphism:

$$
(\phi \star \psi)(f)=(\phi \otimes \psi) \mu^{*}(f), \quad f \in \mathcal{O}_{G, e}
$$

where $\mu$ denotes the multiplication in the supergroup $G$ and $\mu^{*}$ the corresponding sheaf morphism.

The following proposition is a straightforward check.

Proposition 2.8. The convolution product makes $D(G, e)$ into an associative superalgebra, its unit being the evaluation at e, denoted by $\mathrm{ev}_{e}: \mathrm{O}_{G, e} \rightarrow k$.

We now want to examine the relation of $D(G, e)$ with the universal enveloping superalgebra of the supergroup $G$. Since $D(G, e) \supset D_{1}(G, e)^{+} \cong \operatorname{Lie}(G)$, by the universal property of the universal enveloping superalgebra $U(\mathfrak{g})$, we have a superalgebra morphism $\alpha: \mathcal{U}(\mathfrak{g}) \rightarrow D(G, e)$.

Observation 2.9. If $G$ is an algebraic supergroup and the characteristic of $k$ is positive, say char $(k)=p>0$, then $D(G, e)$ contains more than the elements coming from $U(\mathfrak{g})$ (refer to Example 2.3). This is because the divided powers $X^{m} / m$ ! are in $D(G, e)$ but not in $U(\mathfrak{g})$. Again similarly, as in the classical situation, we have that any morphism $U(\mathfrak{g}) \rightarrow D(G, e)$ factors via the universal enveloping restricted algebra $u^{r}(\mathfrak{g})$ :

$$
u(\mathfrak{g}) \rightarrow u^{r}(\mathfrak{g})=u(\mathfrak{g}) /\left(X^{p}-X^{[p]}\right) \rightarrow D(G, e)
$$

where $X^{[p]}$ denotes the derivation in $\mathfrak{g}$ corresponding to $p$-times the derivation $X$ (which is a derivation here, since we are in characteristic $p$ ).

Let $\operatorname{char}(k)=0$.

Proposition 2.10. The morphism $\alpha: \mathcal{U}(\mathfrak{g}) \rightarrow D(G, e)$ is an isomorphism. 
Proof. This is done essentially in the same way as in the classical setting, which is detailed in [Varadarajan 2004, Chapter I] for the analytic category and [Demazure and Gabriel 1970, Chapter II, 6, 1.1] for the algebraic category.

Proposition 2.11. There is an isomorphism of the superalgebra of distributions on a supergroup $G$ and the superalgebra of the left-invariant differential operators on $G$. In this situation $U(\mathfrak{g})$ is isomorphic to the superalgebra of the left-invariant differential operators on $G$.

Proof. The same remarks as in the previous proof apply.

The distributions of an affine algebraic supergroup. We now want to restrict ourselves to the case of affine algebraic supergroups. As we shall see, this algebraic setting shares many similarities with the differential one.

Consider the module of distributions $D(G)$ (see Observation 2.2):

$$
D(G)=\bigcup_{x \in G(k)} D(G, x) \subset \mathcal{O}(G)^{*} .
$$

Definition 2.12. If $\phi=\sum \phi_{p_{i}}$ is a distribution with $\phi_{p_{i}} \in D\left(G, p_{i}\right)$ we say that $\phi$ is supported at $\left\{p_{i}\right\}$. On the whole $D(G)$ we have a well-defined associative product, called the convolution product:

$$
\left(\phi_{p} \star \phi_{q}\right)(f)=\left(\phi_{p} \otimes \phi_{q}\right) \mu^{*}(f)
$$

and its unit is $\mathrm{ev}_{e}$, the evaluation at the unit element: $\operatorname{ev}_{e}(f)=f(e)$. Here $\mu^{*}$ denotes (as before) the comultiplication in the Hopf superalgebra $\mathcal{O}(G)$.

Observation 2.13. If $\phi_{p}$ and $\phi_{q}$ are distributions supported at $p$ and $q$ respectively, then $\phi_{p} \star \phi_{q}$ is supported at $p q$. This is a consequence of the fact that

$$
\mu^{*}\left(m_{p q}\right) \subset m_{p} \otimes \mathcal{O}(G)+\mathcal{O}(G) \otimes m_{q}
$$

where $m_{x}$ is as usual the maximal ideal of the sections in $\mathcal{O}(G)$ vanishing at $x \in G(k) . m_{x}=m_{x, 0}+J_{O(G)}$, that is, $m_{x}$ is the sum of $m_{x, 0}$ the ordinary maximal ideal corresponding to the topological rational point $x \in G(k)$ and the ideal $J_{\mathscr{C}(G)}$ generated by the odd sections in $\mathcal{O}(G)$.

Lemma 2.14. Let $\phi_{g} \in D(G, g)$. Then there exists a unique $\phi_{e} \in D(G, e)$ such that $\phi_{e}=\mathrm{ev}_{g^{-1}} \star \phi_{g}$.

Proof. Since $\phi_{g}=\left(\mathrm{ev}_{g} \star \mathrm{ev}_{g^{-1}}\right) \star \phi_{g}$, define $\phi_{e}=\mathrm{ev}_{g^{-1}} \star \phi_{g} \in D(G, e)$.

Proposition 2.15. $D(G)$ is a super Hopf algebra with comultiplication $\Delta$, counit $\epsilon$ and antipode $S$ given by

$\Delta\left(\phi_{g}\right)(f \otimes g):=\phi_{g}(f \cdot g), \quad \epsilon\left(\phi_{g}\right)(f):=\phi_{g}\left(\mathrm{ev}_{e}(f)\right), \quad S\left(\phi_{g}\right)(f):=\phi_{g}\left(i^{*}(f)\right)$, where $i: G \rightarrow G$ denotes the inverse morphism. 
Proof. Direct check.

Let $k|G|$ be the group algebra corresponding to the ordinary group $G(k)$, i.e.,

$$
k|G|=\left\{\sum_{\substack{g \in G(k) \\ \lambda_{g} \in k}} \lambda_{g} g\right\} .
$$

Proposition 2.16. We have a linear isomorphism

$$
\Psi: D(G) \rightarrow k|G| \otimes \mathcal{U}(g), \quad \phi_{g} \mapsto g \otimes \phi_{e},
$$

which endows $k|G| \otimes U(\mathfrak{g})$ of a Hopf superalgebra structure. This structure is induced by the natural Hopf structures on the group algebra $k|G|$ and $U(\mathfrak{g})$ :

$$
\Delta_{k|G|}(g)=g \otimes g, \quad \Delta_{U(\mathfrak{g})}(U)=U \otimes 1+1 \otimes U, \quad g \in G(k), U \in \mathfrak{g} .
$$

The superalgebra structure is defined by

$$
(g \otimes X)(h \otimes Y)=g h \otimes\left(h^{-1} X\right) Y, \quad g \in G(k), \quad X, Y \in \mathcal{U}(\mathfrak{g}),
$$

with $h^{-1} X:=\mathrm{ev}_{h^{-1}} \star X \star \mathrm{ev}_{h}$. (By Proposition 2.10 we identify distributions at $e$ with elements in $U(\mathfrak{g})$.)

Proof. This is done with a direct check. We just point out that it is enough to do such check just on generators.

\section{Super Harish-Chandra pairs}

The theory of super Harish-Chandra Pairs (SHCP) that we shall develop presently provides an equivalent way to approach the analytic or affine algebraic supergroups.

Definition of an SHCP. Any time we say supergroup we mean an analytic or an affine algebraic supergroup over a field $k$ of characteristic zero.

Definition 3.1. Let $G_{0}$ be a group (complex analytic or affine algebraic) and $\mathfrak{g}$ a super Lie algebra. We make the following assumptions:

(1) $\mathfrak{g}_{0} \simeq \operatorname{Lie}\left(G_{0}\right)$.

(2) $G_{0}$ acts on $\mathfrak{g}$ and this action restricted to $\mathfrak{g}_{0}$ is the adjoint representation of $G_{0}$ on $\operatorname{Lie}\left(G_{0}\right)$. Moreover, the differential of the action is the Lie bracket. We denote such an action by Ad or as $g . X, g \in G_{0}, X \in \mathfrak{g}$.

Then $\left(G_{0}, \mathfrak{g}\right)$ is called a super Harish-Chandra pair $(S H C P)$.

A morphism of SHCP is simply a pair of morphisms $\psi=\left(\psi_{0}, \rho^{\psi}\right)$ preserving the SHCP structure; that is:

(1) $\psi_{0}: G_{0} \rightarrow H_{0}$ is a group morphism (in the analytic or algebraic category).

(2) $\rho^{\psi}: \mathfrak{g} \rightarrow \mathfrak{h}$ is a super Lie algebra morphism. 
(3) $\psi_{0}$ and $\rho^{\psi}$ are compatible in the sense that $\rho_{{\mid g_{0}}^{\psi}}^{\psi}=d \psi_{0}$ and

$$
\operatorname{Ad}\left(\psi_{0}(g)\right) \circ \rho^{\psi}=\rho^{\psi} \circ \operatorname{Ad}(g) .
$$

When $G_{0}$ is an analytic group we shall speak of an analytic SHCP, when $G_{0}$ is an affine algebraic group of an algebraic SHCP.

We would like to show that the category of (analytic of algebraic) SHCP, denoted by (shcps), is equivalent to the category of supergroups (analytic or algebraic), denoted by (sgrps). In order to do this we start by associating in a natural way a supergroup to an SHCP.

Definition 3.2. Let $\left(G_{0}, \mathfrak{g}\right)$ be an SHCP. The sheaf $\mathscr{O}_{G_{0}}$ of the ordinary group $G_{0}$ carries a natural action of $U\left(\mathfrak{g}_{0}\right)$, since the elements of $U\left(\mathfrak{g}_{0}\right)$ act on the sections in $\mathcal{O}_{G_{0}}(U)$ as left-invariant differential operators. We define $\mathcal{O}_{G}(U)$ as

$$
\mathcal{O}_{G}(U):=\operatorname{Hom}_{U\left(\mathfrak{g}_{0}\right)}\left(\mathcal{U}(\mathfrak{g}), \mathcal{O}_{G_{0}}(U)\right), \quad U \subset_{\text {open }} G_{0} .
$$

Proposition 3.3. The assignment $U \mapsto \mathcal{O}_{G}(U)$ is a sheaf of superalgebras on $G_{0}$, where the superalgebra structure on $\mathrm{O}_{G}(U)$ is given by

$$
f_{1} \cdot f_{2}=m_{\mathscr{O}_{G_{0}}} \circ\left(f_{1} \otimes f_{2}\right) \circ \Delta_{U(\mathfrak{g})}
$$

and the restriction morphisms $\rho_{U V}: \mathscr{O}_{G}(U) \rightarrow \sigma_{G}(V)$ are $\rho_{U V}(f):=\tilde{\rho}_{U V} \circ f$, where $\tilde{\rho}_{U V}$ are the restrictions of the ordinary sheaf $\mathscr{O}_{G_{0}}$.

Proof. The check $f_{1} \cdot f_{2}$ is an associative product is routine, while the sheaf property comes from the fact $O_{G_{0}}$ is an ordinary sheaf.

We now show that $\left(G_{0}, \mathscr{O}_{G}\right)$ is a superspace, by showing that is globally split; in other words, that

$$
\mathcal{O}_{G}(U) \cong \mathscr{O}_{G_{0}}(U) \otimes \wedge\left(\mathfrak{g}_{1}\right) .
$$

Theorem 3.4. (1) Let $\gamma: \bigwedge\left(\mathfrak{g}_{1}\right) \rightarrow \mathcal{U}(\mathfrak{g})$ be the symmetrization map, given by

$$
\gamma\left(X_{1} \wedge \cdots \wedge X_{p}\right)=\frac{1}{p !} \sum_{\tau \in S_{p}}(-1)^{|\tau|} X_{\tau(1)} \cdots X_{\tau(p)},
$$

where $|\tau|$ denotes the parity of the permutation $\tau$. Then

$$
\widehat{\gamma}: \mathcal{U}\left(\mathfrak{g}_{0}\right) \otimes \wedge\left(\mathfrak{g}_{1}\right) \rightarrow \mathcal{U}(\mathfrak{g}), \quad X \otimes Y \mapsto X \cdot \gamma(Y)
$$

is an isomorphism of super left $\mathcal{U}\left(\mathfrak{g}_{0}\right)$-modules.

(2) $\left(G_{0}, O_{G}\right)$ is globally split; i.e., for each open subset $U \subseteq G_{0}$ there is an isomorphism of superalgebras

$$
\mathfrak{O}_{G}(U) \simeq \operatorname{Hom}\left(\bigwedge\left(\mathfrak{g}_{1}\right), \mathscr{O}_{G_{0}}(U)\right) \simeq \mathscr{O}_{G_{0}}(U) \otimes \wedge\left(\mathfrak{g}_{1}\right)^{*} .
$$

Hence $\mathrm{O}_{G}$ carries a natural $\mathbb{Z}$-gradation. 
Proof. (1) is an application of Poincaré-Birkhoff-Witt (PBW) theorem (see [Varadarajan 2004]), while for (2) consider the map

$$
\phi_{U}: \mathscr{O}_{G}(U) \rightarrow \operatorname{Hom}\left(\bigwedge\left(\mathfrak{g}_{1}\right), \mathscr{O}_{G_{0}}(U)\right), \quad f \mapsto f \circ \gamma .
$$

Since $\gamma$ is a supercoalgebra morphism, $\phi_{U}$ is a superalgebra morphism. In fact, $\phi_{U}\left(f_{1} \cdot f_{2}\right)=m \circ f_{1} \otimes f_{2} \circ \Delta_{U(\mathfrak{g})} \circ \gamma=m \circ f_{1} \otimes f_{2} \circ(\gamma \otimes \gamma) \Delta_{U(\mathfrak{g})}=\phi_{U}\left(f_{1}\right) \phi_{U}\left(f_{2}\right)$.

That $\phi_{U}$ is a superalgebra isomorphism follows at once from $U\left(\mathfrak{g}_{0}\right)$-linearity.

As an almost immediate consequence of the previous theorem we have:

Corollary 3.5. If $G_{0}$ is an analytic manifold or algebraic scheme, then $\left(G_{0}, O_{G}\right)$ is a superspace.

In the next sections we complete the task of showing $\left(G_{0}, O_{G}\right)$ is a supergroup by providing explicit expression for the multiplication, unit and inverse. This will lead to the main result of the paper, namely the equivalence of categories between the SHCP and supergroups. We now state the main result of the paper and then we shall prove it with different methods in the next sections, since at this point the analytic and algebraic categories diverge and require dramatically different treatment.

Theorem 3.6. Let $k$ be a field of characteristic zero, $k=\mathbb{C}$ if we are in the algebraic category. Define the functors

$$
\begin{array}{clc}
\mathscr{H}: \text { (sgrps) } & \rightarrow \text { (shcps) } \\
G & \mapsto\left(G_{0}, \operatorname{Lie}(G)\right) \\
\phi & \mapsto\left(|\phi|,(d \phi)_{e}\right)
\end{array}
$$

and

$$
\begin{array}{cccc}
\mathscr{K}:(\text { shcps }) & \rightarrow & \text { (sgrps) } \\
\left(G_{0}, \mathfrak{g}\right) & \mapsto & \bar{G}:=\left(G_{0}, \operatorname{Hom}_{\left(\mathfrak{g}_{0}\right)}\left(\mathcal{U}(\mathfrak{g}), \bigcirc_{G_{0}}\right)\right) \\
\psi=\left(\psi_{0}, \rho^{\psi}\right) & \mapsto & f \mapsto \psi_{0}^{*} \circ f \circ \rho_{\psi},
\end{array}
$$

where $G$ and $\left(G_{0}, \mathfrak{g}\right)$ are objects and $\phi, \psi$ are morphisms of the corresponding categories (in the definition of $\mathcal{H}, G_{0}$ is the ordinary group underlying $G$ ). Then $\mathscr{H}$ and $\mathscr{H}$ define an equivalence between the categories of supergroups (analytic or algebraic) and super Harish-Chandra pairs (analytic or algebraic).

Analytic SHCP. Let $k=\mathbb{C}$.

For analytic SHCP it is relatively easy to define a supergroup structure on the superspace $\left(G_{0}, O_{G}\right)$ we have defined above, by mimicking what happens in the smooth case. In fact for an analytic ordinary group $G_{0}$, the action of $U\left(\mathfrak{g}_{0}\right)$ on $\mathscr{O}_{G_{0}}$ is given by

$$
\left(\tilde{D}_{Z} \cdot f\right)(g)=f\left(g e^{t Z}\right), \quad Z \in \mathfrak{g}_{0}, \quad f \in \mathbb{O}_{G_{0}}(U),
$$


where $e^{t Z}$ denotes the one-parameter subgroup corresponding to the element $Z \in \mathfrak{g}_{0}$. Notice that at this point we encounter an important difference with the algebraic setting, since in that case we do not have a result such as the Frobenius theorem available.

Proposition 3.7. $\left(G_{0}, O_{G}\right)$ is an analytic supergroup where the multiplication $\mu$, inverse $i$ and unit e are defined via the corresponding sheaf morphisms by

$$
\begin{aligned}
{\left[\mu^{*}(f)(X, Y)\right](g, h) } & =\left[f\left(\left(h^{-1} \cdot X\right) Y\right)\right](g h), \\
{\left[i^{*}(f)(X)\right]\left(g^{-1}\right) } & =\left[f\left(g^{-1} \cdot \bar{X}\right)\right](g), \\
e^{*}(f) & =[f(1)](e),
\end{aligned}
$$

for $f \in \mathrm{O}_{G}(U)$ and $g, h \in|G|$, where $|G|$ is the topological space underlying $G_{0}$. Here $\bar{X}$ denotes the antipode in $\mathcal{U}(\mathfrak{g})$.

Note. We shall discuss the peculiar form of $\mu^{*}, i^{*}, e^{*}$ in Remark 3.14.

Proof. The proof of this result is the same as in the differential smooth setting, where everything is defined in the same way (see [Carmeli et al. 2011, Chapter 7]. In particular to prove that $\mu^{*}, i^{*}, e^{*}$ are $U\left(\mathfrak{g}_{0}\right)$-morphisms is harder than the verification of the compatibility conditions and the Hopf superalgebra properties. As an example, let us verify $\mu$ is well-defined the other properties being essentially the same type of calculation. Due to the PBW theorem, it is enough to prove $\mathfrak{g}_{0}$-linearity. Let $Z \in \mathfrak{g}_{0} ;$ then

$$
\begin{aligned}
\mu^{*}(f)(Z X, Y)(g, h) & =f\left(h^{-1}(Z X) Y\right)(g h) \\
& =f\left(\left(h^{-1} \cdot Z\right)\left(h^{-1} \cdot X\right) Y\right)(g h) \\
& =\tilde{D}_{h^{-1} \cdot Z}\left[f\left(\left(h^{-1} \cdot X\right) Y\right)\right](g h) .
\end{aligned}
$$

On other hand,

$$
\begin{aligned}
{\left[\left(\tilde{D}_{Z} \otimes \mathrm{id}\right)\left(\mu^{*}(f)(X, Y)\right)\right](g, h) } & =\frac{d}{d t}{ }_{\mid t=0} f\left(\left(h^{-1} X\right) Y\right)\left(g e^{t Z} h\right) \\
& =\frac{d}{d t}{ }_{\mid t=0} f\left(\left(h^{-1} X\right) Y\right)\left(g h e^{t\left(h^{-1} Z\right)}\right) \\
& =\tilde{D}_{h^{-1} Z}\left[f\left(\left(h^{-1} \cdot X\right) Y\right)\right](g h) .
\end{aligned}
$$

Similarly, for the left entry, one finds

$$
\begin{aligned}
\mu^{*}(f)(X, Z Y)(g, h) & =f\left(\left(h^{-1} X\right) Z Y\right)(g h) \\
& =f\left(Z\left(h^{-1} X\right) Y+\left[h^{-1} X, Z\right] Y\right)(g h) \\
& =\tilde{D}_{Z}\left(f\left(\left(h^{-1} X\right) Y\right)\right)(g h)+f\left(\left[h^{-1} X, Z\right] Y\right)(g h)
\end{aligned}
$$

and 


$$
\begin{aligned}
\frac{d}{d t}_{\mid t=0} \mu^{*}(f)(X, Y)\left(g, h e^{t Z}\right) & =\frac{d}{d t}{ }_{\mid t=0} f\left(\left(\left(h e^{t Z}\right)^{-1} X\right) Y\right)\left(g h e^{t Z}\right) \\
& =\left[\tilde{D}_{Z} f\left(\left(h^{-1} X\right) Y\right)\right](g h)+f\left(\left[\left(h^{-1} X\right), Z\right] Y\right)(g h) .
\end{aligned}
$$

We are now ready for the proof of Theorem 3.6 in the analytic setting.

Theorem 3.8. There is an equivalence of categories between analytic SHCP and analytic supergroups expressed by the functors $\mathscr{H}$ and $\mathscr{H}$ in Theorem 3.6.

Proof. Let us first show the correspondence between morphisms. If $\phi$ is a morphisms of analytic supergroups, it is immediate that $\left(|\phi|,(d \phi)_{e}\right)$ is a morphism of SHCP. Conversely, if $\psi=\left(\psi_{0}, \rho_{\psi}\right)$ is a morphism of $\operatorname{SHCP}\left(G_{0}, \mathfrak{g}\right),\left(H_{0}, \mathfrak{h}\right)$, then the map $\psi^{*}: \mathrm{O}_{H}(U) \rightarrow \mathrm{O}_{G}\left(\psi_{0}^{-1}(U)\right)$ defined by $\psi^{*}(f)=\psi_{0}^{*} \circ f \circ \rho_{\psi}$ is a sheaf morphism and $\left(\psi_{0}, \psi^{*}\right)$ is a morphism of the supergroups $G$ and $H$. As one can check, the assignments in Theorem 3.6 establish a one-to-one correspondence between the set of morphisms of SHCPs and the set of morphisms of analytic supergroups.

We now turn to the correspondence between the objects. Let $G$ be a supergroup and $\bar{G}$ the supergroup obtained from the $\operatorname{SHCP}\left(G_{0}\right.$, Lie $\left.(G)\right)$, where $G_{0}$ is the ordinary analytic group underlying $G$. As for the smooth setting, let us define the morphism $\eta: \bar{G} \rightarrow G$ by

$$
\begin{aligned}
\eta^{*}: \mathbb{O}_{G}(U) & \rightarrow \mathbb{O}_{\bar{G}}(U)=\operatorname{Hom}_{U\left(\mathfrak{g}_{0}\right)}\left(U(\mathfrak{g}), \mathscr{O}_{G_{0}}(U)\right), \\
s & \mapsto\left(\bar{s}: X \rightarrow(-1)^{|X|}\left|\left(D_{X} s\right)\right|\right) .
\end{aligned}
$$

Here $D_{X}$ denotes the left-invariant differential operator on $G$ associated with $X \in U(\mathfrak{g})$, that is $D_{X}=(1 \otimes X) \mu^{*}$. The definition is well-posed as one can directly check, moreover $\eta$ is a SLG morphism, i.e.,

$$
\eta \circ \mu_{\bar{G}}=\mu_{G} \circ(\eta \times \eta) .
$$

Indeed, for each $s \in \mathcal{O}(G), X, Y \in \mathcal{U}(\mathfrak{g})$, and $g, h \in G_{0}$,

$$
\begin{aligned}
{\left[\left(\left(\eta^{*} \otimes \eta^{*}\right) \mu_{G}^{*}(s)\right)(X, Y)\right](g, h) } & =(-1)^{|X|+|Y|}\left|\left(D_{X} \otimes D_{Y}\right) \mu_{G}^{*}(s)\right|(g, h) \\
& =(-1)^{|X|+|Y|}\left|D_{h^{-1} \cdot X} D_{Y} s\right|(g h) \\
& =\left[\eta^{*}(s)\left(\left(h^{-1} \cdot X\right) Y\right)\right](g h) \\
& =\left[\left(\mu_{\bar{G}}^{*} \eta^{*}(s)\right)(X, Y)\right](g, h) .
\end{aligned}
$$

The last thing to check is that $\eta$ is an isomorphism. This is true because $|\eta|$ is clearly bijective and, for each $g \in G_{0}$, the differential $(d \eta)_{g}$ is bijective:

$$
\begin{aligned}
{\left[(d \eta)_{g}\left(\bar{D}_{X g}\right)\right](s) } & =\bar{D}_{X g} \eta^{*}(s)=\operatorname{ev}_{g}\left(\bar{D}_{X} \eta^{*}(s)\right)=\left[\bar{D}_{X} \eta^{*}(s)\right](1)(g) \\
& =(-1)^{|X|} \eta^{*}(s)(X)(g)=\left|\left(D_{X} s\right)\right|(g)=D_{X g}(s),
\end{aligned}
$$


where we denote by $\bar{D}_{X}$ a left-invariant differential operator on $\bar{G}$ corresponding to $X \in \mathcal{U}(\mathfrak{g})$ while $D_{X}$ denotes a left-invariant differential operator on $G$.

We conclude using the inverse function theorem, which holds also for analytic supermanifolds and again this is an important difference with the algebraic setting, where we do not have this tool available.

Remark 3.9 ( $p$-adic SHCP). One can define $p$-adic supermanifolds, supergroups and SHCP through the obvious same definitions within the framework described classically in [Serre 1992]. In fact since the category of $p$-adic manifolds resembles very closely the category of analytic manifolds, it is then only reasonable to expect that one can develop along the same lines the theory of $p$-adic supermanifolds. Once the basic results, like the inverse function theorem, are established, the equivalence of categories between $p$-adic supergroups and the $p$-adic SHCP will then follow through the same proof we have detailed for the analytic category.

Algebraic SHCP. We now prove our main result, Theorem 3.6, in the case of $G$ an affine algebraic supergroup over an algebraically closed field of characteristic zero.

The category of affine algebraic supergroups is equivalent to the category of commutative Hopf superalgebras; hence we need to show that there is a unique commutative Hopf superalgebra $\mathrm{O}(G)$ associated to a $\operatorname{SHCP}\left(G_{0}, \mathfrak{g}\right)$, namely the superalgebra of the global sections of the sheaf $O_{G}$ as in Definition 3.2.

Since the exponential appears for the action of $\mathcal{U}\left(\mathfrak{g}_{0}\right)$ on $\mathcal{O}\left(G_{0}\right)$ (see beginning of previous subsection), the question is entirely classical and it is treated in detail in [Demazure and Gabriel 1970, Chapter 2] for the algebraic setting. We shall briefly review a few key facts, sending the reader to that reference for details.

Let $G_{0}$ be an algebraic group and $A$ a commutative algebra, $p: A(t) \rightarrow A[t] /\left(t^{2}\right)$ the natural projection, $t$ even. By definition, $\operatorname{Lie}\left(G_{0}\right)(A)=\operatorname{ker} G_{0}(p)$. Since $G_{0}$ is affine we have $G_{0} \subset \mathrm{GL}(V)$ for a suitable vector space $V$; hence we can write

$$
\begin{aligned}
\operatorname{Lie}\left(G_{0}\right)(A) & =\{1+t Z\} \subset G_{0}(A(t)) \subset \operatorname{GL}(V)(A(t)) \\
& =\operatorname{GL}(V)(A)+t \operatorname{End}(V)(A)
\end{aligned}
$$

for suitable $Z \in \operatorname{End}(V)(A)$, where $\operatorname{End}(V)$ is the functor of points of the superscheme of the endomorphisms of the vector space $V$. Very often $\operatorname{Lie}\left(G_{0}\right)$ is identified with the subspace in $\operatorname{End}(V)$ consisting of the elements $Z$. As a notation device we define

$$
e^{t Z}=1+t Z \in G_{0}(A(t)) .
$$

Let $g \in G_{0}(A)=\operatorname{Hom}\left(\mathbb{O}\left(G_{0}\right), A\right)$, that is, $g$ is an $A$-point of $G_{0}$, and let $f \in \mathbb{O}\left(G_{0}\right)$. As another common notational device, we denote $g(f)$ with $f(g)$. Since $A$ embeds naturally in $A(t)$ we can view $g$ also as an $A(t)$-point of $G_{0}$ and consider $f\left(g e^{t Z}\right)$. 
We then define

$$
\frac{d}{d t}_{\mid t=0} f\left(g e^{t Z}\right)=b,
$$

where $f\left(g e^{t Z}\right)=\left(g e^{t Z}\right)(f)=a+b t \in A(t)$. One sees that the left-hand side of (*) corresponds to the natural action of $Z \in \operatorname{Lie}\left(G_{0}\right)$ on $\mathcal{O}\left(G_{0}\right)$ via left-invariant operators, that is,

$$
\frac{d}{d t}{ }_{\mid t=0} f\left(g e^{t Z}\right)=(1 \otimes Z) \mu^{*}(f),
$$

which we denoted by $\tilde{D}_{Z} f$ in the analytic category.

We now go back to the super setting and prove the analogue of Proposition 3.7.

Proposition 3.10. The superalgebra $\mathcal{O}(G)=\operatorname{Hom}\left(\mathcal{U}(\mathfrak{g}), \mathcal{O}\left(G_{0}\right)\right)$ associated to the algebraic $\operatorname{SHCP}\left(G_{0}, \mathfrak{g}\right)$ is an Hopf superalgebra where the comultiplication $\mu^{*}$, antipode $i^{*}$ and counit $e^{* 3}$ are defined as follows:

$$
\begin{aligned}
{\left[\mu^{*}(f)(X, Y)\right](g, h) } & =\left[f\left(\left(h^{-1} \cdot X\right) Y\right)\right](g h), \\
{\left[i^{*}(f)(X)\right]\left(g^{-1}\right) } & =\left[f\left(g^{-1} \cdot \bar{X}\right)\right](g), \\
e^{*}(f) & =[f(1)](e),
\end{aligned}
$$

for $f \in \mathcal{O}(G), g, h \in|G|$. Here $\bar{X}$ denotes the antipode in $\mathcal{U}(\mathfrak{g})$.

Proof. It is the same as for Proposition 3.7. Though the context is different, once the exponential terminology assumes a meaning for the algebraic category, the calculations are the same.

The next proposition shows a very natural fact: given an SHCP $\left(G_{0}, \mathscr{O}_{G}\right)$, the sheaf $O_{G}$ is the structural sheaf associated with the superalgebra of its global sections $\mathcal{O}(G)$, so that the morphisms $\mu^{*}, i^{*}, e^{*}$ are actually defined as the appropriate sheaf morphisms, corresponding to $\mu, i, e$, multiplication, inverse and unit in the algebraic supergroup $G=\underline{\operatorname{Spec}} \mathcal{O}(G)$. corresponding to the $\operatorname{SHCP}\left(G_{0}, \mathfrak{g}\right)$.

Proposition 3.11. Let $\left(G_{0}, \mathfrak{g}\right)$ be an $S H C P$, with $G_{0}$ an affine group scheme and let $O_{G}$ as in 3.1. Then $G:=\left(G_{0}, O_{G}\right)$ is a supergroup scheme.

Proof. In Proposition 3.10 we have seen that $\mathcal{O}(G):=\operatorname{Hom}_{u\left(\mathfrak{g}_{0}\right)}\left(U(\mathfrak{g}), \mathcal{O}_{G_{0}}\left(G_{0}\right)\right)$ has an Hopf superalgebra structure, moreover by Theorem 3.4 it is globally split. Hence we only need to prove that $G=\underline{\operatorname{Spec}} \mathcal{O}(G)$. Clearly the topological spaces underlying the superspaces $G=\left(G_{0}, \mathscr{O}_{G}\right)$ and $\operatorname{Spec} O(G)$ are homeomorphic. We only need to show that $\mathscr{O}_{\mathcal{O}(G)} \cong \mathcal{O}_{G}$, where $\mathscr{O}_{\mathcal{O}(G)}$ denotes the structural sheaf associated with the superring $\mathcal{O}(G)$. We set up a morphism

\footnotetext{
${ }^{3}$ In analogy with Proposition 3.7 we have kept the terminology $\mu^{*}, i^{*}, e^{*}$, though we are not making (yet) any claim on the sheaf morphisms.
} 


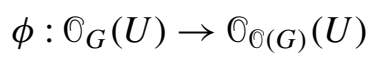

taking $s: \mathcal{U}(\mathfrak{g}) \rightarrow \mathscr{O}_{G_{0}}(U)$ to

$$
\phi(s): U \rightarrow \coprod_{x \in U} \mathrm{O}(G)_{x},
$$

as follows. Any $s \in \mathbb{O}_{G}(U)$ gives raise naturally to $s_{x}: \mathcal{U}(\mathfrak{g}) \rightarrow \mathbb{O}_{G_{0}}(U) \rightarrow \mathbb{O}_{G_{0}, x}$. Since as a $U\left(\mathfrak{g}_{0}\right)$ module, $\mathcal{U}(\mathfrak{g})$ is finitely generated, say by $N$ generators, once we fix those generators, $s_{x}$ is equivalent to the choice of $N$ elements in $\mathbb{O}_{G_{0}, x}$. Since likewise $O(G)_{x}$ is finitely generated by $N$ elements as free $O_{G_{0}, x}$-module (those $N$ elements corresponds dually to the generators of $U(\mathfrak{g})$ as $U\left(\mathfrak{g}_{0}\right)$-module), we have that $s_{x}$ can be viewed as an element of $\mathcal{O}(G)_{x}$. So we define

$$
\phi(s)(x)=s_{x}, \quad x \in U .
$$

We leave to the reader the check that $\phi$ is a sheaf isomorphism.

Theorem 3.12. The category of algebraic SHCP is equivalent to the category of affine algebraic supergroups.

Proof. We need to establish a one to one correspondence between the objects and the morphisms.

As for the objects, if $\left(G_{0}, \mathfrak{g}\right)$ is an algebraic SHCP, we can define an affine algebraic supergroup defining the following Hopf superalgebra (see Proposition 3.10):

$$
\mathcal{O}\left(G_{0}, \mathfrak{g}\right)=\underline{\operatorname{Hom}}_{U\left(\mathfrak{g}_{0}\right)}\left(U(\mathfrak{g}), \mathcal{O}\left(G_{0}\right)\right) .
$$

Conversely, if we have an algebraic supergroup, we can find right away the SHCP associated to it. What we need to show is that these operations are one the inverse of the other; that is,

$$
\mathcal{O}\left(G_{0}, \mathfrak{g}\right) \cong \mathcal{O}(G)
$$

where $G_{0}$ is the algebraic group underlying $G$ and $\mathfrak{g}=\operatorname{Lie}(G)$. Certainly they are isomorphic as $\mathcal{O}\left(G_{0}\right)$-modules, since they have the same reduced part and, by a result from [Masuoka 2005], they both can be written as $O\left(G_{0}\right) \otimes \Lambda$ for some exterior algebra $\Lambda$, but being their odd dimension the same, the two exterior algebras are isomorphic.

We can set a map

$$
\eta^{*}: \mathcal{O}(G) \rightarrow \mathcal{O}\left(G_{0}, \mathfrak{g}\right)
$$

taking $s$ to $\bar{s}: X \mapsto(-1)^{|X|}\left|D_{X}(s)\right|$, where $D_{X}(s)=(1 \otimes X) \mu^{*}$. This is a welldefined morphism of Hopf superalgebras and $X \mapsto(-1)^{|X|}\left|D_{X}(s)\right|$ is a $U\left(\mathfrak{g}_{0}\right)$ morphism. This is done precisely in the same way as in the proof of Theorem 3.8.

We now want to show that $\eta^{*}$ is surjective. This will imply that $\eta^{*}$ is an isomorphism. In fact the two given supergroups $G=\underline{\operatorname{Spec}} \mathcal{O}(G)$ and $\bar{G}=\underline{\operatorname{Spec}} \mathcal{O}\left(G_{0}, \mathfrak{g}\right)$ 
are smooth superschemes, with the same underlying topological space and same Lie superalgebra (hence the same superdimension), and $\eta^{*}$ induces an injective morphism $\eta: \bar{G} \rightarrow G$ (see [Fioresi and Gavarini 2013, Section 2]).

For the surjectivity of $\eta^{*}$, we need to show that, for each morphism of $U\left(\mathfrak{g}_{0}\right)$ modules $\bar{s}: \mathcal{U}(\mathfrak{g}) \rightarrow \mathcal{O}\left(G_{0}\right)$, there exists $s \in \mathcal{O}(G)$ such that $\bar{s}(X)=(-1)^{|X|}\left|D_{X}(s)\right|$. Since $U(\mathfrak{g}) \cong U\left(\mathfrak{g}_{0}\right) \otimes \wedge\left(\mathfrak{g}_{1}\right)$ (see Theorem 3.4) and $\bar{s}$ is a morphism of $U\left(\mathfrak{g}_{0}\right)$ modules, $\bar{s}$ is determined by $\bar{s}\left(\gamma\left(X^{I}\right)\right)$ for $X^{I}=X_{1}^{i_{1}} \ldots X_{n}^{i_{n}}$, where the $X_{i}$ form a basis for $\mathfrak{g}_{1}$ and $i_{j}=0,1$ (again refer to Theorem 3.4). Notice that $X_{i}=\gamma\left(X_{i}\right)$. Since $X_{1}, \ldots, X_{n}$ are linearly independent, also the corresponding left-invariant vector fields $D_{X_{1}}, \ldots, D_{X_{n}}$ will be linearly independent at each point. Let $D_{\gamma(X)}$ denote the left-invariant differential operator corresponding to $\gamma(X) \in \mathcal{U}(\mathfrak{g})$. Notice that fixing a suitable basis in $U(\mathfrak{g})$, the linear morphism $X \mapsto \gamma(X)$ corresponds to an upper triangular matrix and sends linearly independent vectors to linearly independent vectors. Consider the equation $(-1)^{\left|X^{I}\right|}\left|D_{\gamma\left(X^{I}\right)} s\right|=\bar{s}\left(X^{I}\right)$, for $X^{I}=X_{1}^{i_{1}} \ldots X_{n}{ }^{i_{n}}$ a monomial in $\bigwedge\left(\mathfrak{g}_{1}\right)$. This is an equation where each $D_{X_{i}}$ appearing in the expression for $D_{\gamma\left(X^{I}\right)}$ can be expressed as

$$
D_{X_{i}}=\sum a_{i} \partial_{x_{i j}}, \quad p\left(a_{i}\right) \neq p\left(x_{i j}\right)
$$

where the $x_{i j}$ are global coordinates on $\mathrm{GL}_{m \mid n} \supset G$ (regardless of their parity).

Since the $D_{X_{1}}^{i_{1}} \ldots D_{X_{n}}^{i_{n}}$ are linearly independent by the PBW theorem (see also Proposition 2.11), the $D_{\gamma(X)}$ will also be linearly independent, and the equality

$$
(-1)^{|X|}\left|D_{\gamma\left(X^{I}\right)}\right|=\bar{s}\left(X^{I}\right)
$$

will yield a solution

$$
\partial_{x_{i_{1} j_{1}}} \ldots \partial_{x_{i_{r} j_{r}}} s=a_{i_{1} j_{1} \ldots i_{r} j_{r}}
$$

for all $i_{1} j_{1} \ldots i_{r} j_{r}$ such that

$$
s=\sum a_{i_{1} j_{1} \ldots i_{r} j_{r}} x_{i_{1} j_{i}} \ldots x_{i_{r} j_{r}} .
$$

We leave to the reader the correspondence between morphisms.

Example 3.13. We want to verify explicitly the surjectivity of $\eta^{*}$ in the case of GL(1|1) and make a few remarks on how to extend the calculation to the case of $G=\mathrm{GL}(m \mid n)$. Let $\mathcal{O}(\mathrm{GL}(1 \mid 1))=k\left[a_{11}, a_{22}, \alpha_{12}, \alpha_{21}\right]\left[a_{11}^{-1}, a_{22}^{-1}\right]$. Let

$$
\begin{aligned}
& D_{12}=\left(1 \otimes \partial_{\alpha_{12}}\right) \mu^{*}=a_{11} \partial_{\alpha_{12}}+\alpha_{21} \partial_{a_{22}}, \\
& D_{21}=\left(1 \otimes \partial_{\alpha_{21}}\right) \mu^{*}=\alpha_{12} \partial_{a_{11}}+a_{22} \partial_{\alpha_{21}},
\end{aligned}
$$

be the left-invariant vector fields corresponding to the generators $\partial_{\alpha_{12}}, \partial_{\alpha_{21}}$ of $\operatorname{Lie}(G)_{1}$; then 


$$
\begin{aligned}
\gamma\left(D_{12} D_{21}\right)= & \frac{1}{2}\left(D_{12} D_{21}-D_{21} D_{12}\right) \\
= & \frac{1}{2}\left(a_{11} \partial_{a_{11}}-a_{22} \partial_{a_{22}}\right) \\
& +a_{11} a_{22} \partial_{\alpha_{12}} \partial_{\alpha_{21}}+\text { terms with coefficients in } J_{\overparen{O}(\mathrm{GL}(1 \mid 1)),}
\end{aligned}
$$

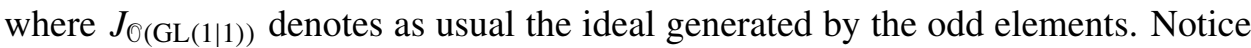
that the terms with coefficients in $J_{\mathscr{O}(\mathrm{GL}(1 \mid 1))}$ do not contribute in the expression $\left|D_{\gamma\left(D_{12} D_{21}\right)} s\right|$. For the same reason, the term $a_{11} \partial_{a_{11}}-a_{22} \partial_{a_{22}}$ will make a contribution only if applied to $s^{0}$, and consequently can be considered not as unknown, but as a known term. This is important in case one wants to generalize this procedure to $\mathrm{GL}(m \mid n)$; in fact only the terms containing only odd derivations will produce new quantities to be determined.

Given $\bar{s}: \mathcal{U}(\mathfrak{g}) \rightarrow \mathcal{O}\left(G_{0}\right)$ we want to determine $s \in \mathcal{O}(G)$, with $\eta^{*}(s)=\bar{s}$. Since $\operatorname{Lie}\left(\mathrm{GL}(1 \mid 1)_{1}=\left\langle\partial_{\alpha_{12}}, \partial_{\alpha_{21}}\right\rangle\right.$, the map $\bar{s}$ is determined once we know its image on $\wedge \operatorname{Lie}\left(\mathrm{GL}(1 \mid 1)_{1}\right.$, that is,

$$
s^{0}=\bar{s}(1), \quad s^{12}=\bar{s}\left(\partial_{\alpha_{12}}\right), \quad s^{21}=\bar{s}\left(\partial_{\alpha_{21}}\right), \quad s^{12,21}=\bar{s}\left(\gamma\left(\partial_{\alpha_{12}} \partial_{\alpha_{21}}\right)\right) .
$$

Consequently the $s$ we want to determine must satisfy the equations

$$
\begin{aligned}
s^{0} & =|1 s|, \\
s^{12} & =-\left|a_{11} \partial_{\alpha_{12}} s+\alpha_{21} \partial_{a_{22}} s\right|, \\
s^{21} & =-\left|\alpha_{12} \partial_{a_{11}} s+a_{22} \partial_{\alpha_{21}} s\right|, \\
s^{12,21} & =\left|\frac{1}{2}\left(a_{11} \partial_{a_{11}} s-a_{22} \partial_{a_{22}} s\right)+a_{11} a_{22} \partial_{\alpha_{12}} \partial_{\alpha_{21}} s\right| .
\end{aligned}
$$

A simple calculation gives us

$$
s=s^{0}+\frac{\alpha_{12} s^{12}}{a_{11}}-\frac{\alpha_{21} s^{21}}{a_{22}}+\left[s^{12,21}-\frac{1}{2}\left(a_{11} \partial_{a_{11}} s^{0}-a_{22} \partial_{a_{22}} s^{0}\right)\right] \frac{\alpha_{12} \alpha_{21}}{a_{11} a_{22}} .
$$

There is no conceptual obstacle to extending this calculation to the case of $G=\mathrm{GL}(m \mid n)$. If $\mathrm{O}(G)=k\left[a_{i j}, \alpha_{k l}\right]\left[d_{1}^{-1}, d_{2}^{-1}\right]$ where $d_{1}=\operatorname{det}\left(a_{i j}\right)_{\{1 \leq i, j \leq m\}}$ and $d_{2}=\operatorname{det}\left(a_{i j}\right)_{\{m+1 \leq i, j \leq m+n\}}$, the left-invariant vector fields are given by

$$
X_{i j}=\left(1 \otimes \partial_{x_{i j}}\right) \mu^{*}=\sum_{k} x_{k i} \partial_{x_{k j}},
$$

where $x_{i j}$ denote the coordinates on $\mathrm{GL}(m \mid n)$ regardless of their parity. We can then repeat the calculation we did above. Notice that any even derivation appearing in the expression $\left|D_{\gamma(X)} s\right|$ will affect only $s^{0}=|1 s|$ since we are taking the reduction modulo the ideal of the odd nilpotents.

Remark 3.14. We clarify the relation between the Hopf superalgebra $O(G)=$ $\operatorname{Hom}\left(\mathcal{U}(\mathfrak{g}), \mathcal{O}\left(G_{0}\right)\right)$ associated to the $\operatorname{SHCP}\left(G_{0}, \mathfrak{g}\right)$ and the distribution superalgebra $D(G)$ of the supergroup $G$ (also naturally associated to the same SHCP). 
For an affine supergroup $G$, the superalgebra of distributions $D(G)$ has a natural Hopf superalgebra structure; see Proposition 2.15. This structure is inherited by $k|G| \otimes U(\mathfrak{g})$ through the linear isomorphism with $D(G)$ given in Proposition 2.16. The superalgebra of global sections of $G, \mathcal{O}(G)=\operatorname{Hom}\left(\mathcal{U}(\mathfrak{g}), \mathcal{O}\left(G_{0}\right)\right)$ can then be naturally viewed as a subspace of $D(G)^{*} \cong(k|G| \otimes U(\mathfrak{g}))^{*}$, since elements in $\mathbb{O}(G)$ arise as suitable morphisms $|G| \times \mathcal{U}(\mathfrak{g}) \rightarrow k$. One can then immediately verify that the Hopf superalgebra structure on $\mathcal{O}(G) \subset D(G)^{*}$ is precisely obtained by duality, from the Hopf superalgebra on $D(G)$ suitably restricting the comultiplication, counit and antipode morphisms.

\section{Action of supergroups and SHCPs}

We now want to relate the action of an analytic of algebraic supergroup $G$ on a supermanifold or superscheme $M$, with the action of the corresponding SHCP $\left(G_{0}, \mathfrak{g}\right)$ on $M$. In this section, if $g \in|G|$ we denote by $\hat{g}: \mathbb{C}^{0 \mid 0} \rightarrow G$ the morphism whose pull-back is the evaluation at $g$. We recall a well-know definition:

Definition 4.1. A morphism $a: G \times M \rightarrow M$ is called an action of $G$ on $M$ if

$$
a \circ\left(\mu \times \mathbb{1}_{M}\right)=a \circ\left(\mathbb{1}_{G} \times a\right)
$$

and

$$
a \circ\left\langle\hat{e}, \mathbb{1}_{M}\right\rangle=\mathbb{1}_{M} .
$$

In the functor of points notation, this is the same as demanding the following, where $T$ is a supermanifold (resp. a superscheme) and $M(T)=\operatorname{Hom}(T, M)$ are the $T$-points of $M$ :

(1) $1 \cdot x=x$ for all $x \in M(T)$, where 1 the unit in $G(T)$.

(2) $\left(g_{1} g_{2}\right) \cdot x=g_{1} \cdot\left(g_{2} \cdot x\right)$ for all $x \in M(T)$ and all $g_{1}, g_{2} \in G(T)$.

Here, as usual, we are writing $a(g, x)$ as $g \cdot x$.

If an action $a$ of $G$ on $M$ is given, then we say that $G$ acts on $M$.

Definition 4.2. An action of an analytic $\operatorname{SHCP}\left(G_{0}, \mathfrak{g}\right)$ on a supermanifold $M$ consists of an action

$$
\underline{a}: G_{0} \times M \rightarrow M
$$

of the reduced Lie group $G_{0}$ on $M$, with $\underline{a}: a \circ\left(j_{|G| \rightarrow G} \times \mathbb{1}_{M}\right)$, plus a representation

$$
\begin{aligned}
\rho_{a}: \mathfrak{g} & \rightarrow \operatorname{Vec}(M)^{\mathrm{op}} \\
X & \mapsto\left(X \otimes \mathbb{1}_{\mathcal{O}(M)}\right) a^{*}
\end{aligned}
$$


of the super Lie algebra $\mathfrak{g}$ of $G$ on the opposite of the Lie superalgebra of vector fields over $M$, the whole satisfying the compatibility relations

$$
\begin{aligned}
\left.\rho_{a}\right|_{\mathfrak{g}_{0}}(X) & =\left(X \otimes \mathbb{1}_{\mathcal{O}(M)}\right) \underline{a}^{*} & & \text { for all } X \in \mathfrak{g}_{0}, \\
\rho_{a}(g . Y) & =\left(\underline{a}^{g^{-1}}\right)^{*} \rho_{a}(Y)\left(\underline{a}^{g}\right)^{*} & & \text { for all } g \in|G|, Y \in \mathfrak{g},
\end{aligned}
$$

where $a^{g}: M \rightarrow M$ is given by $a^{g}:=a \circ\left\langle\hat{g}, \mathbb{1}_{M}\right\rangle$.

The next proposition tells us that actions of an SHCP correspond bijectively to actions of the corresponding analytic supergroup.

Proposition 4.3. Let $G$ be an analytic supergroup acting on a supermanifold $M$. Then there is an action of the $\operatorname{SHCP}\left(G_{0}, \operatorname{Lie}(G)\right)$ on $M$. Conversely, given an action of the $\operatorname{SHCP}\left(G_{0}, \mathfrak{g}\right)$ on $M$, there is a unique action $a_{\rho}: G \times M \rightarrow M$ of the analytic supergroup $G$ corresponding to the given $S H C P$ on $M$ whose reduced and infinitesimal actions are the given ones. If $U$ is an open subset of $M$, we have

$$
\begin{aligned}
a_{\rho}^{*}: \mathscr{O}_{M}(U) & \rightarrow \operatorname{Hom}_{U\left(\mathfrak{g}_{0}\right)}\left(\mathcal{U}(\mathfrak{g}),\left(\mathbb{O}_{G_{0}} \hat{\otimes} \mathscr{O}_{M}\right)\left(|a|^{-1}(U)\right)\right), \\
f & \mapsto\left[X \mapsto(-1)^{|X|}\left(\mathbb{1}_{\mathcal{O}\left(G_{0}\right)} \otimes \rho(X)\right) \underline{a}^{*}(f)\right] .
\end{aligned}
$$

Proof. Let us check that $a_{\rho}^{*}(f)$ is $\mathcal{U}\left(\mathfrak{g}_{0}\right)$-linear. For all $X \in \mathcal{U}(\mathfrak{g})$ and $Z \in \mathfrak{g}_{0}$ we have

$$
\begin{aligned}
a_{\rho}^{*}(f)(Z X) & =(-1)^{|X|}(\mathbb{1} \otimes \rho(Z X)) \underline{a}^{*}(f) \\
& =(-1)^{|X|}(\mathbb{1} \otimes \rho(X))\left(\mathbb{1} \otimes Z_{e} \otimes \mathbb{1}\right)\left(\mathbb{1} \otimes \underline{a}^{*}\right) \underline{a}^{*}(f) \\
& =(-1)^{|X|}(\mathbb{1} \otimes \rho(X))\left(\mathbb{1} \otimes Z_{e} \otimes \mathbb{1}\right)\left(\tilde{\mu}^{*} \otimes \mathbb{1}\right) \underline{a}^{*}(f) \\
& =\left(\tilde{D}_{Z} \otimes \mathbb{1}\right)\left[a_{\rho}^{*}(f)(X)\right] .
\end{aligned}
$$

We now check that $a_{\rho}^{*}$ is a superalgebra morphism.

$$
\begin{aligned}
{\left[a_{\rho}^{*}\left(f_{1}\right) \cdot a_{\rho}^{*}\left(f_{2}\right)\right](X) } & =m_{\mathscr{O}_{G_{0}} \hat{\otimes} \mathscr{O}_{M}}\left[a^{*}\left(f_{1}\right) \otimes a^{*}\left(f_{2}\right)\right] \Delta(X) \\
& =(-1)^{|X|} m\left[\left(\mathbb{1} \otimes \rho\left(X_{(1)}\right)\right) \underline{a}^{*}\left(f_{1}\right) \otimes\left(\mathbb{1} \otimes \rho\left(X_{(2)}\right)\right) \underline{a}^{*}\left(f_{2}\right)\right] \\
& =(-1)^{|X|}(\mathbb{1} \otimes \rho(X))\left(\underline{a}^{*}\left(f_{1}\right) \cdot \underline{a}^{*}\left(f_{2}\right)\right)=a_{\rho}^{*}\left(f_{1} \cdot f_{2}\right)(X),
\end{aligned}
$$

where $f_{i} \in \mathcal{O}(M)$ and $X_{(1)} \otimes X_{(2)}$ denotes $\Delta(X)$. Concerning the "associative" property, we have that, for $X, Y \in \mathcal{U}(\mathfrak{g})$ and $g, h \in G_{0}$,

$$
\begin{aligned}
{\left[\left(\mu^{*} \otimes \mathbb{1}\right) a_{\rho}^{*}(f)\right](X, Y)(g, h) } & =\left[a_{\rho}^{*}(f)\right]\left(h^{-1} \cdot X Y\right)(g h) \\
& =(-1)^{|X|+|Y|+|X||Y|} \rho(Y) \rho\left(h^{-1} \cdot X\right)\left(\underline{a}^{g h}\right)^{*}(f) \\
& =(-1)^{|X|+|Y|+|X||Y|} \rho(Y)\left(\underline{a}^{h}\right)^{*} \rho(X)\left(\underline{a}^{g}\right)^{*}(f) \\
& =\left[\left(\mathbb{1} \otimes a_{\rho}^{*}\right) a_{\rho}^{*}(f)\right](X, Y)(g, h),
\end{aligned}
$$


and, finally, $\left(\mathrm{ev}_{e} \otimes \mathbb{1}\right) a_{\rho}^{*}(f)=\rho(1)=f$.

Uniqueness can be proved as follows. Let $a$ be an action of $G$ on $M$ and let $\left(\underline{a}, \rho_{a}\right)$ be as in Proposition 4.3. If $f \in \mathcal{O}_{M}(U)$, then

$$
\begin{aligned}
a^{*}(f) & \in\left(\operatorname{Hom}_{U\left(\mathfrak{g}_{0}\right)}\left(u(\mathfrak{g}), \mathscr{O}_{G_{0}}\right) \hat{\otimes} \mathcal{O}_{M}\right)\left(|a|^{-1}(U)\right) \\
& \cong \operatorname{Hom}_{U\left(\mathfrak{g}_{0}\right)}\left(u(\mathfrak{g}),\left(\mathcal{O}_{G_{0}} \hat{\otimes} \mathcal{O}_{M}\right)\left(|a|^{-1}(U)\right)\right) ;
\end{aligned}
$$

hence, using $(* *)$ in Definition 4.1 and the fact that $\rho_{a}$ is an antihomomorphism, we obtain for all $X \in \mathcal{U}(\mathfrak{g})$

$$
\begin{aligned}
a^{*}(f)(X) & =(-1)^{|X|}\left[\left(D_{X} \otimes \mathbb{1}\right) a^{*}(\phi)\right](1) \\
& =(-1)^{|X|}\left(\mathbb{1} \otimes \rho_{a}(X)\right)\left(a^{*}(f)(1)\right)=(-1)^{|X|}\left(\mathbb{1} \otimes \rho_{a}(X)\right) \underline{a}^{*}(f) .
\end{aligned}
$$

Let us now assume $G$ is an affine algebraic supergroup over a field of characteristic zero and $\left(G_{0}, \mathfrak{g}\right)$ is the corresponding SHCP and furthermore assume they are acting on a supervariety $M$, the Definition 4.2 being the same, taking the morphisms in the appropriate category.

We state the analogue of the Proposition 4.3 in the algebraic setting, its proof being essentially the same.

Proposition 4.4. Let $G$ be an algebraic supergroup acting on a supervariety $M$ (not necessarily affine). Then there is an action of the $\operatorname{SHCP}\left(G_{0}, \operatorname{Lie}(G)\right)$ on $M$. Conversely, given an algebraic action of the algebraic $\operatorname{SHCP}\left(G_{0}, \mathfrak{g}\right)$ on $M$, there is a unique action $a_{\rho}: G \times M \rightarrow M$ of the algebraic supergroup $G$ corresponding to the given SHCP on $M$ whose reduced and infinitesimal actions are the given ones. If $U$ is an open subset of $M$, we have

$$
\begin{aligned}
a_{\rho}^{*}: \mathscr{O}_{M}(U) & \rightarrow \operatorname{Hom}_{\left(\mathfrak{g}_{0}\right)}\left(\mathcal{(}(\mathfrak{g}),\left(\mathscr{O}_{G_{0}} \otimes \mathscr{O}_{M}\right)\left(|a|^{-1}(U)\right)\right), \\
f & \mapsto\left[X \mapsto(-1)^{|X|}\left(\mathbb{1}_{\mathscr{O}\left(G_{0}\right)} \otimes \rho(X)\right) \underline{a}^{*}(f)\right] .
\end{aligned}
$$

\section{Acknowledgements}

We wish to thank Professor Varadarajan for suggesting the problem and Professors Cassinelli and Gavarini for helpful discussions.

\section{References}

[Balduzzi et al. 2009] L. Balduzzi, C. Carmeli, and G. Cassinelli, "Super G-spaces", pp. 159-176 in Symmetry in mathematics and physics, edited by D. Babbitt et al., Contemp. Math. 490, Amer. Math. Soc., Providence, RI, 2009. MR 2010k:58011 Zbl 1236.58012

[Carmeli et al. 2011] C. Carmeli, L. Caston, and R. Fioresi, Mathematical foundations of supersymmetry, European Mathematical Society, Zürich, 2011. MR 2012h:58010 Zbl 1226.58003 
[Deligne and Morgan 1999] P. Deligne and J. W. Morgan, "Notes on supersymmetry (following Joseph Bernstein)", pp. 41-97 in Quantum fields and strings: a course for mathematicians (Princeton, NJ, 1996/1997), vol. 1, edited by P. Deligne et al., Amer. Math. Soc., Providence, RI, 1999. MR 2001g:58007 Zbl 1170.58302

[Demazure and Gabriel 1970] M. Demazure and P. Gabriel, Groupes algébriques, I: Géométrie algébrique, généralités, groupes commutatifs, Masson, Paris, 1970. MR 46 \#1800 Zbl 0203.23401

[Dieudonné 1970] J. Dieudonné, Éléments d'analyse, Tome III: Chapitres XVI et XVII, Cahiers Scientifiques 33, Gauthier-Villars, Paris, 1970. MR 42 \#5266 Zbl 0208.31802

[Fioresi and Gavarini 2011] R. Fioresi and F. Gavarini, "On the construction of Chevalley supergroups", pp. 101-123 in Supersymmetry in mathematics and physics, edited by S. Ferrara et al., Lecture Notes in Math. 2027, Springer, Berlin, 2011. MR 2906339 Zbl 06078896

[Fioresi and Gavarini 2012] R. Fioresi and F. Gavarini, Chevalley supergroups, Mem. Amer. Math. Soc. 1014, Amer. Math. Soc., Providence, RI, 2012. MR 2918543 Zbl 1239.14045

[Fioresi and Gavarini 2013] R. Fioresi and F. Gavarini, "Algebraic supergroups with Lie superalgebras of classical type", Journal of Lie Group Theory 23:1 (2013), 143-158.

[Jantzen 2003] J. C. Jantzen, Representations of algebraic groups, 2nd ed., Mathematical Surveys and Monographs 107, Amer. Math. Soc., Providence, RI, 2003. MR 2004h:20061 Zbl 1034.20041

[Kostant 1977] B. Kostant, "Graded manifolds, graded Lie theory, and prequantization”, pp. 177-306 in Differential geometrical methods in mathematical physics (Bonn, 1975), edited by K. Bleuler and A. Reetz, Lecture Notes in Math 570, Springer, Berlin, 1977. MR 58 \#28326 Zbl 0358.53024

[Koszul 1983] J.-L. Koszul, "Graded manifolds and graded Lie algebras", pp. 71-84 in Proceedings of the international meeting on geometry and physics (Florence, 1982), edited by M. Modugno, Pitagora, Bologna, 1983. MR 85m:58019 Zbl 0548.22012

[Manin 1988] Y. I. Manin, Gauge field theory and complex geometry, Grundlehren der Mathematischen Wissenschaften 289, Springer, Berlin, 1988. MR 89d:32001 Zbl 0641.53001

[Masuoka 2005] A. Masuoka, "The fundamental correspondences in super affine groups and super formal groups", J. Pure Appl. Algebra 202:1-3 (2005), 284-312. MR 2006e:16066 Zbl 1078.16045

[Masuoka 2012] A. Masuoka, "Harish-Chandra pairs for algebraic affine supergroup schemes over an arbitrary field", Transform. Groups 17:4 (2012), 1085-1121. MR 3000482

[Serre 1992] J.-P. Serre, Lie algebras and Lie groups, 2nd ed., Lecture Notes in Mathematics 1500, Springer, Berlin, 1992. MR 93h:17001 Zbl 0742.17008

[Varadarajan 2004] V. S. Varadarajan, Supersymmetry for mathematicians: an introduction, Courant Lecture Notes in Mathematics 11, New York University, 2004. MR 2005g:58011 Zbl 1142.58009

[Vishnyakova 2011] E. G. Vishnyakova, "On complex Lie supergroups and split homogeneous supermanifolds”, Transform. Groups 16:1 (2011), 265-285. MR 2012b:58010 Zbl 1218.22013 
Received September 5, 2012. Revised September 19, 2012.

Claudio Carmeli

D.I.M.E., UNIVERSITÀ DI GENOVA

VIA CADORNA 2

I-17100 SAVONA

ITALY

and

INFN, SEZIONE DI GENOVA

Via Dodecaneso 33

I-16416 GENOVA

ITALY

claudio.carmeli@gmail.com

RITA FIORESI

DiPARTIMENTO DI MATEMATICA

UNIVERSITÀ DI BOLOGNA

PIAZZA DI PORTA S. DONATO, 5

40126 BOLOGNA

ITALY

fioresi@dm.unibo.it 


\title{
PACIFIC JOURNAL OF MATHEMATICS
}

\author{
msp.org/pjm
}

Founded in 1951 by E. F. Beckenbach (1906-1982) and F. Wolf (1904-1989)

\section{EDITORS}

V. S. Varadarajan (Managing Editor)

Department of Mathematics

University of California

Los Angeles, CA 90095-1555

pacific@math.ucla.edu

Paul Balmer

Department of Mathematics

University of California

Los Angeles, CA 90095-1555

balmer@math.ucla.edu

Daryl Cooper

Department of Mathematics

University of California

Santa Barbara, CA 93106-3080 cooper@math.ucsb.edu

Jiang-Hua $\mathrm{Lu}$

Department of Mathematics

The University of Hong Kong

Pokfulam Rd., Hong Kong jhlu@maths.hku.hk
Don Blasius

Department of Mathematics University of California

Los Angeles, CA 90095-1555

blasius@math.ucla.edu

Robert Finn

Department of Mathematics Stanford University

Stanford, CA 94305-2125

finn@math.stanford.edu

Sorin Popa

Department of Mathematics

University of California

Los Angeles, CA 90095-1555

popa@math.ucla.edu

Paul Yang

Department of Mathematics

Princeton University

Princeton NJ 08544-1000

yang@math.princeton.edu

\section{PRODUCTION}

Silvio Levy, Scientific Editor, production@msp.org

\section{SUPPORTING INSTITUTIONS}

ACADEMIA SINICA, TAIPEI

CALIFORNIA INST. OF TECHNOLOGY

INST. DE MATEMÁTICA PURA E APLICADA

KEIO UNIVERSITY

MATH. SCIENCES RESEARCH INSTITUTE

NEW MEXICO STATE UNIV.

OREGON STATE UNIV.

\author{
STANFORD UNIVERSITY \\ UNIV. OF BRITISH COLUMBIA \\ UNIV. OF CALIFORNIA, BERKELEY \\ UNIV. OF CALIFORNIA, DAVIS \\ UNIV. OF CALIFORNIA, LOS ANGELES \\ UNIV. OF CALIFORNIA, RIVERSIDE \\ UNIV. OF CALIFORNIA, SAN DIEGO \\ UNIV. OF CALIF., SANTA BARBARA
}

\author{
Vyjayanthi Chari \\ Department of Mathematics \\ University of California \\ Riverside, CA 92521-0135 \\ chari@math.ucr.edu \\ Kefeng Liu \\ Department of Mathematics \\ University of California \\ Los Angeles, CA 90095-1555 \\ liu@math.ucla.edu \\ Jie Qing \\ Department of Mathematics \\ University of California \\ Santa Cruz, CA 95064 \\ qing@cats.ucsc.edu
}

These supporting institutions contribute to the cost of publication of this Journal, but they are not owners or publishers and have no responsibility for its contents or policies.

See inside back cover or msp.org/pjm for submission instructions.

The subscription price for 2013 is US \$400/year for the electronic version, and \$485/year for print and electronic.

Subscriptions, requests for back issues and changes of subscribers address should be sent to Pacific Journal of Mathematics, P.O. Box 4163, Berkeley, CA 94704-0163, U.S.A. The Pacific Journal of Mathematics is indexed by Mathematical Reviews, Zentralblatt MATH, PASCAL CNRS Index, Referativnyi Zhurnal, Current Mathematical Publications and the Science Citation Index.

The Pacific Journal of Mathematics (ISSN 0030-8730) at the University of California, c/o Department of Mathematics, 798 Evans Hall \#3840, Berkeley, CA 94720-3840, is published monthly except July and August. Periodical rate postage paid at Berkeley, CA 94704, and additional mailing offices. POSTMASTER: send address changes to Pacific Journal of Mathematics, P.O. Box 4163, Berkeley, CA 94704-0163.

PJM peer review and production are managed by EditFLOW ${ }^{\circledR}$ from Mathematical Sciences Publishers.

PUBLISHED BY

mathematical sciences publishers

nonprofit scientific publishing

http://msp.org/

(C) 2013 Mathematical Sciences Publishers 


\section{PACIFIC JOURNAL OF MATHEMATICS}

Volume $263 \quad$ No. $1 \quad$ May 2013

Biharmonic hypersurfaces in complete Riemannian manifolds

Luis J. Alías, S. Carolina García-Martínez and Marco

RIGOLI

Half-commutative orthogonal Hopf algebras

JULIEN BiCHON and MiCHEL DUBOIS-ViOLETTE

Superdistributions, analytic and algebraic super Harish-Chandra pairs

Claudio CARMELI and RITA FIORESI

Orbifolds with signature $\left(0 ; k, k^{n-1}, k^{n}, k^{n}\right)$

Angel Carocca, Rubén A. Hidalgo and Rubí E.

RODRÍGUEZ

Explicit isogeny theorems for Drinfeld modules

IMIN CHEN and YOONJIN LEE

Topological pressures for $\epsilon$-stable and stable sets

XiAnfeng Ma and ERCAi Chen

Lipschitz and bilipschitz maps on Carnot groups

WILLIAM MEYERSON

Geometric inequalities in Carnot groups

FRANCESCOPAOLO MONTEFALCONE

Fixed points of endomorphisms of virtually free groups

PEDRO V. SILVA

The sharp lower bound for the first positive eigenvalue of the

Folland-Stein operator on a closed pseudohermitian $(2 n+1)$-manifold

CHIN-TUNG Wu

Remark on "Maximal functions on the unit $n$-sphere" by Peter M. Knopf 253 (1987)

HONG-QUAN LI 